\title{
Alcuin contre Théodulphe : un conflit producteur de normes
}

\section{Hélène Noizet}

\section{(2) OpenEdition}

Journals

Édition électronique

URL : http://journals.openedition.org/abpo/1222

DOI : 10.4000/abpo.1222

ISBN : 978-2-7535-1495-9

ISSN : $2108-6443$

\section{Éditeur}

Presses universitaires de Rennes

\section{Édition imprimée}

Date de publication : 20 septembre 2004

Pagination : 113-129

ISBN : 978-2-7535-0053-2

ISSN : 0399-0826

\section{Référence électronique}

Hélène Noizet, «Alcuin contre Théodulphe : un conflit producteur de normes », Annales de Bretagne et des Pays de l'Ouest [En ligne], 111-3 | 2004, mis en ligne le 20 septembre 2006, consulté le 20 avril 2019. URL : http://journals.openedition.org/abpo/1222 ; DOI : 10.4000/abpo.1222

\section{(c) Presses universitaires de Rennes}




\title{
Alcuin contre Théodulphe : un conflit producteur de normes
}

\author{
Hélène NoIzET \\ ATER d'histoire médiévale, ENS Lyon \\ Laboratoire Archéologie et Territoires, UMR CITERES 7163
}

Le conflit qui a opposé Alcuin, alors abbé de Saint-Martin de Tours, à Théodulfe, évêque d'Orléans, forme un riche dossier qui éclaire non seulement la fin de la vie d'Alcuin, mais également les pratiques juridiques et culturelles de cette époque. L'examen de cette affaire permet de croiser deux types de sources écrites : d'une part, le genre épistolaire, avec les lettres d'Alcuin, et, d'autre part, les sources normatives, comme les capitulaires de Charlemagne. Au moins quatre des lettres contenues dans la correspondance d'Alcuin, éditées par Ernst Dümmler ${ }^{1}$, sont directement liées à ce conflit, qui prend des proportions insoupçonnées, et dans lequel Charlemagne intervient en désapprouvant très nettement Alcuin. Si leurs dates précises sont inconnues, tous les historiens s'accordent à penser qu'elles datent ${ }^{2}$ de la fin de l'année 801 ou de 802 . Les différentes facettes de ce conflit permettent de formuler un certain nombre de remarques sur la fabrication du droit à l'époque carolingienne, ainsi que sur le mouvement appelé renaissance carolingienne, ou encore réforme, renouveau, renovatio ou correctio ${ }^{3}$.

Luitpold Wallach ${ }^{4}$ avait déjà analysé ce conflit afin de rechercher les traces de la réception du droit romain, et notamment du Bréviaire d'Alaric, dans les lettres d'Alcuin et de Charlemagne. Si son travail d'identification des sources législatives ayant inspiré ces écrits constitue toujours un

1. Alc. Ep. 245, 246, 247 et 249, p. 393-404. Les lettres 245, 246 et 249 ont été écrites par Alcuin, tandis que la lettre 247 est la réponse de Charlemagne à Alcuin. Pour des traductions partielles de certains de ces documents, voir : S. ALLOTT, Alcuin of York, ${ }^{\circ} 114$, 115 et 116 p. 120-126 (Alc. Ep. 245, 247 et 249); G. TESSIER, Charlemagne..., p. 393-394 (Alc. Ep. 247); L. WallaCH, Alcuin and Charlemagne..., p. 107-109 (Alc. Ep. 247).

2. E. Dümmler avait ainsi daté ces lettres à partir de l'étude de T. SicKeL, Alcuinstudien... Par la suite, L. Wallach, S. Allott, M. Driscoll, puis M.-H. Jullien adoptent tous cette datation.

3. Pour une approche générale, voir en dernier lieu : Ph. DePREuX, «Ambitions et limites des réformes culturelles... ", p. 721-753.

4. L. WaLlaCH, Alcuin and Charlemagne..., c. 7 et 8, p. 99-140. 
apport précieux à la connaissance du droit et de la société carolingienne, nous nous inscrirons dans une démarche méthodologique située à l'opposé de la sienne : nous ne chercherons pas à examiner si Alcuin connaît et respecte les sources juridiques et législatives sur la question du droit d'asile, mais nous agirons à l'inverse en observant comment de nouvelles règles sont élaborées à partir de ce conflit, soit comment un espace juridique se crée à travers la pratique. L'analyse des relations entre normes et comportements privilégie trop souvent un seul questionnement : comment les normes charpentent les pratiques sociales. Dans le cadre de la renaissance carolingienne, l'analyse consiste à montrer que ce mouvement résulte d'une volonté politique des souverains carolingiens et de leurs conseillers ecclésiastiques, la difficulté consistant en la plus ou moins bonne application de ces mesures. Ce dossier permet d'envisager les relations dans l'autre sens, en montrant de quelle manière une norme ou une convention peut être produite à partir de la pratique sociale, en l'occurrence l'élaboration d'un savoir juridique à partir du conflit entre Alcuin et Théodulphe.

\section{Le récit des événements}

À partir de ces quatre lettres de la correspondance d'Alcuin, il est possible de reconstituer le récit détaillé des évènements. Voici comment a débuté toute l'affaire : Théodulphe, élu depuis peu évêque d'Orléans - ce pour quoi Alcuin l'avait d'ailleurs congratulé ${ }^{5}$ - avait jugé un clerc coupable d'un méfait que les sources ne permettent pas de connaître. Alors que ce clerc se trouvait sous la garde de l'évêque, il s'est échappé de sa prison et s'est réfugié dans la basilique de Saint-Martin de Tours afin de bénéficier du droit d'asile. Théodulphe en appelle à Charlemagne, qui l'autorise par un mandement spécial (un indiculus commonitorius d'après Luitpold Wallach) à demander l'extradition du criminel. Des hommes de Théodulfe sont donc venus d'Orléans jusqu'à Tours pour récupérer le fugitif. Mais, alors qu'ils s'apprêtaient à partir, l'annonce d'un piège qui leur aurait été tendu sur la route perturbe le départ. Le criminel profite alors de la confusion pour se réfugier une deuxième fois dans l'église de Saint-Martin. Les hommes de Théodulphe retournèrent donc à Orléans les mains vides. Lorsqu'ils revinrent à Tours, les Orléanais étaient plus nombreux qu'auparavant, ce qui les rend menaçants d'après Alcuin. Le dimanche suivant leur arrivée, huit hommes de Théodulphe, ainsi que l'archevêque de Tours, entrent alors brutalement dans l'église pour récupérer le clerc, alors que des fidèles s'y trouvaient : leur attitude, perçue comme agressive et provocante par les habitants, provoque un tumulte très grave. Ces hommes renversent les chancels qui entouraient l'autel afin de saisir le coupable, qui s'était réfugié entre l'autel et le tombeau de Saint-Martin. C'est alors

5. Théodulphe a été consacré évêque d'Orléans en avril 801. Lettre de félicitations d'Alcuin : Alc. Ep. 225, p. 368. 
que retentit une clameur, entendue par la population, qui se rassembla et entra à son tour dans l'église, pensant que celle-ci était menacée : l'irruption de nombreux pauvres, venus de toute la cité et des environs, armés de bâtons, engendra un chaos indescriptible à l'intérieur de l'église. Si certains frères de Saint-Martin, indignés de l'offense faite à leur saint patron, ont participé à la révolte populaire et ont expulsé manu militari les Orléanais de l'autel, d'autres frères, qui étaient au réfectoire lorsque les cloches sonnèrent, accoururent pour apaiser la révolte. Ceux-ci protégèrent les hommes de Théodulphe de la vindicte populaire en les faisant sortir de l'église pour les amener dans le monasterium.

Théodulphe informa rapidement Charlemagne de l'affaire, en accusant Alcuin et les frères de Saint-Martin d'être passé outre l'ordre donné par Charlemagne et d'avoir provoqué la révolte. Il envoya également une lettre à Alcuin à qui il impute l'entière responsabilité de tout ce désordre. De son côté, Alcuin envoyait à l'empereur sa propre version des faits. Parallèlement, il écrivait deux lettres à ses amis : l'une est destinée à Wizo et Fridugise, qui séjournaient alors à la cour de Charlemagne, l'autre à un évêque inconnu ${ }^{6}$. Dans ses deux lettres, au contenu quasiment identique, il demande à ses amis d'intervenir en sa faveur auprès de Charlemagne, en leur fournissant un appareil de références à la fois scripturaires, juridiques et historiques justifiant son point de vue. Après lecture des deux rapports de Théodulphe et d'Alcuin, Charlemagne fait envoyer une réponse à Alcuin, lettre dans laquelle il ne mâche pas ses mots et qui condamne sans appel Alcuin et les frères de Saint-Martin. Le ton extrêmement sévère de cette lettre est particulièrement impressionnant. Charlemagne reproche ainsi aux frères de Saint-Martin de mener une vie peu conforme à leurs engagements : un des principaux griefs réside dans le fait qu'ils se disent parfois chanoines, parfois moines, parfois ni l'un ni l'autre. Malgré le choix d'un abbé tel qu'Alcuin pour les ramener dans le droit chemin, les frères de Saint-Martin n'ont pas adopté de règle précise et il est difficile de les situer au sein de l'Église. Charlemagne décida d'envoyer un missus, en l'occurrence Teotbert, afin de juger Alcuin et les Tourangeaux sur la charge d'incitation à la révolte. Une fois les jugements prononcés, après un procès de 19 jours, Alcuin envoya un rapport détaillé à Charlemagne, qui avait sans doute reçu celui de Teotbert : Alcuin critique la procédure utilisée par ce dernier et cherche à se justifier et à prouver son innocence.

L'implication d'Alcuin dans cette affaire se mesure au zèle qu'il déploie pour monter un dossier comportant de nombreuses pièces à la fois juridiques et bibliques destinées à servir de preuves.

6. D'après T. Sickel et M. Driscoll, il s'agirait d'Hildebold, archevêque de Cologne, tandis que D. A. Bullough identifiait ce destinataire à Arnon, archevêque de Salzbourg et grand ami d'Alcuin : M.-H. Jullien et F. Perelman, Clavis..., vol. II, p. 315. 


\section{Les sources d'Alcuin}

Dans les deux premières lettres envoyées à ses amis, Alcuin compile et réorganise des éléments tirés de la Bible, ainsi que du droit romain et conciliaire. En bon docteur de la foi, Alcuin donne d'abord ses arguments bibliques, puis les arguments juridiques. Nous avons rassemblé dans le tableau ci-dessous les références scripturaires, essentiellement néo-testamentaires, qu'il utilise dans son argumentaire des deux premières lettres liées à cette affaire. Deux tiers des citations bibliques sont tirées des évangiles de Matthieu et Luc (13 sur 18).

Tableau 1 - Les citations scripturaires dans l'argumentation d'Alcuin

\begin{tabular}{|l|l|l|}
\hline$N^{\circ}$ lettre & Ancien Testament & Nouveau Testament \\
\hline 245 & Ps 22,4 & Mt 5,7 \\
& & Mt 9,13 \\
& & Mt 19,30 \\
& & Mt 20,16 \\
& Mt 27,5 \\
& & Lc 6, 36-37 \\
& & Lc 7, 39 \\
& & Lc 23, 34 \\
& & Lc 23,43 \\
& & I Cor 4,5 \\
& & Ga 6,1 \\
& & He 9,4 \\
& & Ja 2, 13 \\
& & Ja 3,14 \\
& & I Jn $\mathbf{1 , 8}$ \\
\hline 246 & Deut 23, 16-17 & déjà citées dans la lettre \\
& Ez 33, 11-12 & précédente : \\
& & Mt 9,13 \\
& & Lc 6, 37 \\
& & Lc 7, 39 \\
\hline
\end{tabular}

Les quatre premières citations de la lettre $\mathrm{n}^{\circ} 245$ mettent en avant le thème de la clémence, qui se traduit par la nécessité de la miséricorde lors d'un jugement. Alcuin plaide pour un jugement miséricordieux, excluant toute sévérité excessive et permettant au pécheur de se racheter et de retrouver la voie de Dieu. Sur la base des citations néo-testamentaires, qu'il transforme en arguments juridiques, il rappelle que la modération est nécessaire car il ne faut pas se fier aux apparences : les fautes d'un homme, qui semble respectueux de Dieu, peuvent se révéler finalement bien plus graves que celles qui apparaissent évidentes, comme le montre la comparaison entre Juda (Mt 27,5) et la prostituée repentie (Lc 7,39). La double mention du précepte " les premiers seront les derniers " (Mt 19,30 et 20,16) va dans le même sens : Alcuin suggère à mots couverts que Théodulphe, qui est en apparence dans le bon chemin, finira peut-être sa vie dans l'erreur. De plus, Alcuin rappelle que tous les hommes sont pécheurs, y compris les hommes d'Église : si ce coupable est jugé trop sévèrement, alors on ne pourra plus accepter aucun chantre, ni aucun prêtre dans les églises, 
car ils sont tous, à un degré ou à un autre, pécheurs. Alcuin veut montrer que la différence entre le méfait de cet homme et ceux des autres hommes d'Église est une différence de degré et non pas de nature. Enfin, le juge doit être compatissant car seul Dieu est habilité, lors du jugement dernier, à sanctionner sévèrement les coupables (I Cor 4,5). Celui qui reconnaît être coupable mérite la clémence car il pourra, tel le bon larron, accéder au royaume de Dieu (Lc 23, 43).

C'est pourquoi Alcuin insiste sur l'importance de la confession : étant donné que le fugitif s'est confessé à deux prêtres tourangeaux ${ }^{7}$, il mérite que ses juges soient compatissants. Si ses erreurs ne sont pas négligeables, le fait d'avoir reconnu ses erreurs doit être pris en compte, sinon la confession est inutile : au-delà du cas de ce clerc, c'est la validité et la légitimité du sacrement de la pénitence qui est ici en jeu. Pour Alcuin, la confession permet d'effacer ses péchés. Rappelons qu'Alcuin avait déjà écrit des ouvrages fondamentaux sur ces thèmes de la pénitence et de la confession, ouvrages qu'il a vraisemblablement réutilisés pour construire son argumentaire dans ses deux premières lettres. En effet, nous avons souligné en gras dans le tableau ci-dessus les références scripturaires qu'il avait déjà mobilisées dans ses propres écrits pénitentiels, qu'il s'agisse de la lettretraité Ad pueros sancti Martini, récemment édité par Michael Driscoll ${ }^{8}$, ou du traité sur les vertus et les vices ${ }^{9}$ (De virtutibus et vitiis) : ainsi, il apparaît que 4 des 18 références scripturaires citées dans les deux lettres d'Alcuin se trouvaient déjà dans ses œuvres pénitentielles ${ }^{10}$. Nous avons donc la preuve vivante du caractère pratique et concret de ces ouvrages, puisque Alcuin lui-même s'en est resservi pour bâtir son argumentation dans un contexte différent. Il finit sa seconde lettre par une citation vétérotestamentaire qui suggère que le droit d'asile est d'origine biblique (Deut $23,16-17)$, ce qui nous amène au second volet de son argumentation, celui du registre strictement juridique du droit d'asile.

Dans son édition, Ernst Dümmler avait identifié la plupart des sources législatives mentionnées par Alcuin, celle qui restait inconnue ayant été retrouvée par Luitpold Wallach ${ }^{11}$. Le tableau suivant, réalisé à partir des

7. Alc. Ep. 246, p. 399.

8. M. DRISCOLL, "Ad pueros sancti Martini... ", p. 37-59; M. DRISCOLL, Alcuin et la pénitence..., p. 143-166 et annexe 1. Il existe aujourd'hui deux hypothèses pour la datation de cette lettre-traité (Alc. Ep. 131). La proposition initiale de T. Sickel de 796-798 est reprise par M. Driscoll, tandis que D. A. Bullough militait pour une datation plus haute, entre 793/4 et 796/7, avant qu'Alcuin soit abbé de Saint-Martin : D. A. Bullough, "Alcuin and the Kingdom of Heaven..." ", n. 35, p. 214-215.

9. Éd. PL 101, 614-638. Il n'existe pas d'édition critique de cet opuscule. Ce texte correspond à un petit traité de morale, composé vers l'année 801, à la demande d'un ami, Guy de Bretagne, à qui Alcuin le dédie (MGH Ep. 305). Il est destiné à fournir à celui-ci une règle de comportement. Le livre se présente comme un breviarum composé de sermons : il sert d'aide-mémoire et a un caractère pratique.

10. La lettre-traité Ad pueros mentionnait déjà Ez 33, 11-12 et I Jn 1,8, et le traité des vertus et des vices Lc 6, 36 et Mt 5,7. Voir M. Driscoll, Alcuin et la pénitence..., annexe 2, p. 197-200.

11. WALLACH, Alcuin and Charlemagne..., p. 129-130. Deux canons étaient extraits, d'après 
données rassemblées par Luitpold Wallach, rassemble les autorités législatives invoquées par Alcuin en respectant l'ordre qu'il a suivi.

\section{Tableau 2 - Les sources législatives romaines et conciliaires de la défense d'Alcuin}

\begin{tabular}{|c|c|c|}
\hline $\begin{array}{l}\text { Type de } \\
\text { référence }\end{array}$ & Source & Contenu \\
\hline $\begin{array}{l}\text { canon d'un } \\
\text { concile } \\
\text { mérovingien }\end{array}$ & $\begin{array}{l}\text { Concile d'Orléans I } \\
\text { de } 511 \text {, c. } 1\end{array}$ & $\begin{array}{l}\text { Les criminels s'étant réfugiés dans les atria des églises } \\
\text { ou dans la maison épiscopale ne peuvent en être arrachés, } \\
\text { à moins qu'il leur ait été promis par serment prêté sur les } \\
\text { évangiles de ne pas être tué ni mutilé. }\end{array}$ \\
\hline $\begin{array}{l}\text { canon d'un } \\
\text { concile } \\
\text { mérovingien }{ }^{13}\end{array}$ & $\begin{array}{l}\text { Concile d'Orléans IV } \\
\text { de } 541, \text { c. } 21\end{array}$ & $\begin{array}{l}\text { Si quelqu'un se permet d'enlever hors de l'atrium ou } \\
\text { du lieu saint une personne réfugiée dans l'enceinte } \\
\text { de l'église, le réfugié doit être rendu à l'Église et que } \\
\text { l'agresseur fasse réparation en subissant une pénitence. }\end{array}$ \\
\hline $\begin{array}{l}\text { canon d'un } \\
\text { concile } \\
\text { mérovingien } \\
\end{array}$ & $\begin{array}{l}\text { Concile d'Orléans V } \\
\text { de } 549, \text { c. } 22\end{array}$ & $\begin{array}{l}\text { Tout esclave réfugié dans l'enceinte de l'église, une fois } \\
\text { sa faute remise sous serment par son maître, doit être } \\
\text { assuré de son pardon. Si par la suite, le maître châtie } \\
\text { cet homme malgré son serment, ce maître doit être exclu } \\
\text { de la communion de tous. }\end{array}$ \\
\hline $\begin{array}{l}\text { canons } \\
\text { apocryphes d'un } \\
\text { concile } \\
\text { mérovingien }^{15}\end{array}$ & $\begin{array}{l}\text { Collectio Hibernensis, } \\
\text { c. } 28.3 \text { et } 28.9 \mathrm{a}\end{array}$ & $\begin{array}{l}\text { Les meurtriers ne doivent pas être expulsés des portes } \\
\text { de l'église qui les protège de tout type de peine s'ils } \\
\text { donnent satisfaction à la partie offensée. Les fidèles } \\
\text { réfugiés dans le sein de l'église ne doivent pas en être } \\
\text { enlevés facilement, mais ils doivent y être traités correc- } \\
\text { tement jusqu'au jugement. }\end{array}$ \\
\hline $\begin{array}{c}\text { exemple } \\
\text { historique }^{16}\end{array}$ & $\begin{array}{l}\text { loi apocryphe de } \\
\text { Constantin tiré des } \\
\text { Actus Sylvestri, } \\
\text { p. } 513,1.19\end{array}$ & $\begin{array}{l}\text { Le } 5^{\mathrm{e}} \text { jour après son baptême, une église fut construite } \\
\text { à l'endroit où Constantin avait reçu la vertu de la } \\
\text { consécration : lorsqu'un quelconque accusé se réfugiait } \\
\text { dans celle-ci, il devait être protégé par les juges du péril } \\
\text { qui existait jusqu'à présent. }\end{array}$ \\
\hline $\begin{array}{l}\text { canon d'une loi } \\
\text { romaine }^{17}\end{array}$ & $\begin{array}{l}\text { Bréviaire d'Alaric IX, } \\
34,1 \text { (Code Théodo- } \\
\text { sien IX, 45,4), } \\
\text { constitution des } \\
\text { empereurs Theodo- } \\
\text { sius II et Valentinia- } \\
\text { nus III du } 23 \text { mars } 431\end{array}$ & $\begin{array}{l}\text { Personne ne doit porter la main sur une personne réfugiée } \\
\text { dans l'église. Les espaces adjacents aux églises, tels } \\
\text { que les portiques, les atria, les maisons et les places, } \\
\text { doivent accorder la même protection que l'intérieur } \\
\text { des églises, afin que les réfugiés ne restent pas autour } \\
\text { des autels et ne polluent pas les églises. }\end{array}$ \\
\hline
\end{tabular}

Alcuin, du concile d'Agde, alors qu'ils ne figuraient pas dans les décisions prises par ce concile. L. Wallach a montré que ces deux canons ont été en réalité recopiés par Alcuin à partir de la collection canonique Hibernensis, collection composée en Irlande vers 700. Simplement L. Wallach s'est trompé dans la référence qu'il donne : il s'agit du chapitre 28, et non pas 38 .

12. J. GAUDEMET, B. BASDEVANT, Les canons des conciles mérovingiens..., vol. 1, p. 70-73.

13. Ibid., p. 278-279.

14. Ibid., p. 317-319. Alcuin indiquait qu'il s'agissait du canon 21, alors que le texte cité correspond au canon $22: \mathrm{L}$. Wallach avait remarqué que cela prouvait qu'Alcuin avait utilisé la Collectio codicis Laureshamensis, qui intervertit effectivement les canons 21 et 22 .

15. H. WASSERSCHLEBEN, Die irische Kanonensammlung, p. 95-96. Pour plus d'informations sur la Collectio Hibernensis, voir P. FouRnIER et G. LE BRAS, Histoire des collections canoniques en Occident..., vol. 1, p. 62. Voir aussi P. FouRNIER, « De l'influence de la collection irlandaise..." ", p. 27-78.

16. B. Mombritius, Sanctuarium seu Vitae Sanctorum, vol. 2, p. 513, l. 19.

17. G. HAENEL, Lex romana visigothorum, p. 208-210. 


\begin{tabular}{|l|l|l|}
\hline $\begin{array}{l}\text { canon d'une loi } \\
\text { romaine }\end{array}$ & $\begin{array}{l}\text { Bréviaire d'Alaric IX, } \\
2,3 \text { (Code Théodo- } \\
\text { sien IX, 3,7), constitu- } \\
\text { tion des empereurs } \\
\text { Honorius et Theodo- } \\
\text { sius II du 25 janvier 409 }\end{array}$ & $\begin{array}{l}\text { Tous les dimanches, les juges doivent faire sortir les } \\
\text { personnes accusées de leurs prisons, tout en laissant } \\
\text { sous bonne garde, pour qu'elles récupèrent de la } \\
\text { nourriture et aillent aux bains. Si un juge néglige cela, } \\
\text { il devra payer la peine fixée par la loi. }\end{array}$ \\
\hline $\begin{array}{l}\text { canon d'une loi } \\
\text { romaine }\end{array}$ & $\begin{array}{l}\text { Bréviaire d'Alaric, } \\
\text { Pauli Sententice, }, 28.1\end{array}$ & $\begin{array}{l}\text { Toute personne ayant tué, torturé fouetté ou enchainé } \\
\text { un citoyen romain ayant fait appel à l'empereur, ou } \\
\text { ayant ordonné de telles pratiques, sera condamné par la } \\
\text { loi julienne sur la force publique. Les humiliores seront } \\
\text { punis de la peine capitale, tandis que les honestiores } \\
\text { seront déportés sur une île. }\end{array}$ \\
\hline $\begin{array}{l}\text { canon d'une loi } \\
\text { romaine } 20\end{array}$ & $\begin{array}{l}\text { Bréviaire d'Alaric, } \\
\text { Pauli Sententice, v, 28.2. }\end{array}$ & $\begin{array}{l}\text { La loi julienne décrète que tout juge sera accusé de } \\
\text { violence publique s'il est responsable d'outrages du type } \\
\text { mort, torture, flagellation envers un citoyen romain qui } \\
\text { lui avait fait appel ou qui avait demandé à être admis en } \\
\text { présence du princeps. Les humiliores seront punis de la } \\
\text { peine capitale, tandis que les honestiores seront déportés } \\
\text { sur une île. }\end{array}$ \\
\hline $\begin{array}{l}\text { exemple } \\
\text { historique }\end{array}$ & $\begin{array}{l}\text { Paul Orose, Historiae } \\
\text { adversum paganos, } \\
\text { VII, 39.1 }\end{array}$ & $\begin{array}{l}\text { Alaric, qui a assiégé Rome et y a jeté la confusion, avait } \\
\text { cependant ordonné que si des personnes s'étaient } \\
\text { réfugiées dans de saints lieux, notamment les basiliques } \\
\text { des saints apôtres Pierre et Paul, les Wisigoths les laisse- } \\
\text { raient en sécurité sans les molester. }\end{array}$ \\
\hline
\end{tabular}

Il est particulièrement intéressant d'observer qu'Alcuin détourne certains de ces textes en ne les citant pas en entier : il ne conserve que les éléments qui soutiennent sa version des faits, tandis qu'il passe habilement sous silence les parties de textes qui contredisent son point de vue. Seules sont retenues les parties de loi présentant les droits du réfugié, tandis que leurs devoirs ne sont jamais évoqués. Ainsi, Luitpold Wallach ${ }^{22}$ avait observé qu'Alcuin n'utilisait pas la totalité de la sententia 2 du canon $5 \mathrm{du}$ livre 5 du Bréviaire d'Alaric, dont il ne cite que les deux premières phrases. Or, cette sentence empêche les personnes déjà jugées par une cour ou qui auraient déjà avoué leurs crimes, de faire appel à l'empereur ${ }^{23}$ et de bénéficier d'un statut de faveur. Ces personnes, qui sont reconduites en prison, peuvent faire l'objet de châtiments corporels. C'est parce que le verbe confiteri, qui est employé dans la loi romaine au sens juridique de "l'aveu ", est interprété par Alcuin comme la " confession ", que cette loi lui pose un problème et qu'il prend soin de l'écarter. Cette partie de la loi se serait parfaitement appliquée au cas du clerc fugitif, puisqu'il avait déjà été jugé coupable, et empêchait donc ce fugitif de faire appel à l'empereur.

Nous constatons qu'Alcuin a procédé au même " nettoyage " de ces sources en ce qui concerne les conciles mérovingiens. Ainsi, il ne cite pas dans sa totalité le canon 22 du concile d'Orléans v, tenu en 549. Or, la fin de ce canon ${ }^{24}$ autorisait le maître à récupérer son esclave réfugié dans

18. Ibid., p. 174.

19. Ibid., p. 438.

20. Ibid., p. 438.

21. Paul Orose, Histoires (Contre les Païens), vol. 3, p. 113-114.

22. L. WaLlaCh, Alcuin and Charlemagne..., p. 135.

23. G. HAENEL, Lex romana visigothorum, V, 28, 2, p. 438.

24. J. GAUDEMET et B. BASDEVANT, Les canons des conciles mérovingiens..., p. 318-319. 
l'église, à partir du moment où le maître a pardonné sous serment sa faute : si le fugitif refuse de sortir, le maître a le droit de se saisir de lui, afin que le clergé ne soit pas accusé de rétention d'esclaves.

Toutes ces prescriptions visaient à permettre le bon fonctionnement des procédures judiciaires : elles établissent que le droit d'asile ne doit pas constituer un moyen d'échapper à la justice. Selon le Bréviaire d'Alaric et les statuts synodaux mérovingiens, l'asile n'est pas l'équivalent d'une amnistie : ces hommes réfugiés dans l'église doivent être jugés, et s'ils sont reconnus coupables et s'ils ont avoué leurs fautes, leurs maîtres peuvent les récupérer et ils sont passibles des mêmes châtiments que les autres. Ces deux sources comprenaient manifestement deux volets : si elles commencent par énoncer les droits des réfugiés dans l'asile, elle corrige aussitôt en précisant les limites de ce droit : comme Alcuin a systématiquement fait disparaître ce deuxième volet, le droit d'asile qu'il défend ne semble comporter aucune contrainte. En réalité, il en comporte une, qui apparaît essentielle aux yeux d'Alcuin - mais pas aux yeux de Charlemagne - celle de la confession et du repentir : à partir du moment où le réfugié reconnaît ses crimes et fait acte de pénitence, il doit bénéficier du pardon non seulement de Dieu, mais également de la clémence de la justice humaine. C'est parce que la vraie pénitence réside dans le regret des péchés avoués lors de la confession, ainsi que dans le changement de conduite en s'abstenant de commettre à l'avenir ces mêmes péchés, qu'Alcuin réinvente un droit d'asile délaissant l'autorité publique du juge local, au profit du jugement princier lui-même, et favorisant une prise de conscience intérieure de la faute. Le fait, constaté ci-dessus, que ses écrits pénitentiels et cette lettre utilisent les mêmes références scripturaires confirme ce lien établi par Alcuin entre droit d'asile et confession.

Ainsi, Alcuin réélabore le droit romain et mérovingien afin de définir un droit d'asile qui témoigne de sa préoccupation première, celle du salut des hommes, en accordant une place majeure au sacrement de la confession. Il dénature la portée juridique originelle de l'asile chrétien romain pour défendre un droit d'asile, dont il réaffirme l'origine biblique ${ }^{25}$, et qui garantit le recours au jugement de l'empereur pour ceux qui s'y réfugient, sans se soucier ni de leur culpabilité ni des procédures judiciaires, la seule obligation étant celle de la confession. On comprend mieux dès lors pourquoi Charlemagne prend le parti de Théodulphe dans ce conflit : c'est que la version alcuinienne du droit d'asile laisse la porte ouverte à toutes les dérives judiciaires en permettant à de réels criminels d'échapper à la justice. Charlemagne ne raisonne pas comme Alcuin en homme d'église responsable du salut des hommes, mais en homme politique chargé du maintien de la paix publique. Il paraît évident à ce dernier que la confession ne peut suffire à rétablir la concorde entre les deux parties. C'est pourquoi il met un coup d'arrêt à de telles considérations en répondant aussi durement à Alcuin.

25. Fondée principalement sur Deut. 23, 16-17, versets qu'il cite dans sa deuxième lettre (Alc. Ep. 246). 
Outre la divergence de point de vue sur les modalités du retour à la paix (confession et pénitence d'un côté, énonciation de la justice publique de l'autre), un autre aspect spécifique du discours d'Alcuin réside dans la conception singulièrement ecclésiale de l'asile.

\section{Un espace sacré centré sur l'ecclesia au détriment de l'atrium}

Nous avons rassemblé dans le tableau ci-dessous tous les termes topographiques employés par Alcuin lors de la description de la révolte.

Tableau 3 - Le vocabulaire topographique employé par Alcuin lors du récit de la révolte

\begin{tabular}{|l|l|}
\hline$N^{\circ}$ lettre & Extrait de la lettre \\
\hline $\mathrm{n}^{\circ} 245$ & $\begin{array}{l}\text { reus ad ecclesiam sancti Martini confugit } \\
\text { eum dimiserunt ante fores ecclesiae stantem } \\
\text { octo primates homines intraverunt in ecclesiam } \\
\text { illi octo inruentes intra cancellos altaris } \\
\text { quos expulerunt fratres ante faciem altaris } \\
\text { episcopi homines foras ecclesiam expulerunt }\end{array}$ \\
\hline $\mathrm{n}^{\circ} 246$ & $\begin{array}{l}\text { reus ad ecclesiam sancti Martini confugit } \\
\text { eum dimiserunt ante fores ecclesiae stantem } \\
\text { principales homines intraverunt ecclesiam } \\
\text { reum iacentem ante sepulchrum sancti confessoris Christi } \\
\text { principales homines inruentes intra cancellos altaris } \\
\text { quos expulerunt fratres ante faciem altaris } \\
\text { ne inter altare et sepulcrum sanctissimi confessoris Christi sanguinis effu- } \\
\text { sio foret } \\
\text { raptus est homo medio populi ut duceretur foras }\end{array}$ \\
& $\begin{array}{l}\text { tumultu qui ortus est in ecclesia beati Martini vel foras in atrio } \\
\text { pontifex noster nimis inoportune intravit ecclesiam } \\
\text { aliqui inpigri a foris venerunt dum signa sonare audierunt } \\
\text { fratres audientes signa prosiluerunt de refectorio } \\
\text { homines episcopi qui ducti sunt in monasterium ubi salvi esse potuissent }\end{array}$ \\
\hline $\mathrm{n}^{\circ} 249$
\end{tabular}

Le vocabulaire topographique d'Alcuin met très largement en exergue l'ecclesia, terme répété pas moins de 9 fois. À l'intérieur de celle-ci, Alcuin évoque tout particulièrement l'autel (5 mentions) et le sépulcre de Martin (2 mentions). D'après le récit d'Alcuin, autel et reliques sont au même niveau topographique et entourés de chancels. Alcuin prend soin d'insister sur le sacrilège commis par les hommes de Théodulphe qui ont saccagé ces balustrades pour accéder à l'espace compris entre l'autel et le tombeau, où s'était réfugié le criminel. Des frères de Saint-Martin, indignés par l'offense faite à leur saint patron et craignant une effusion de sang à cet endroit, ont expulsé ces hommes hors de ce périmètre sacré, délimité par des cancelli, qui définissent un pôle de sacralité à l'intérieur de l'ecclesia.

Les deux mentions des portes de l'église (fores ecclesiae) peuvent être rapprochées des quatre occurrences de l'adverbe foris ou foras, qui renvoie à l'extérieur de l'église, mais aussi au monde extérieur d'une façon plus générale. Ces termes se rapportent à une opposition dedans/dehors : 
tout ce qui se situe au-delà des portes de l'église semble renvoyer au monde extérieur.

À l'inverse, il est frappant qu'Alcuin n'emploie quasiment jamais les mots désignant l'espace protégé par le droit d'asile. D'après les documents juridiques mobilisés par Alcuin, celui-ci correspond à l'église ainsi qu'à l'espace immédiatement environnant, souvent enclos et incluant plusieurs types de places ou d'édifices (portiques, maisons). Lorsqu'il écrit son propre texte, Alcuin n'exploite pas la très grande variété des expressions mentionnées dans les sources tardo-antiques pour désigner l'asile, expressions qu'il a tout de même recopiées lorsqu'il cite ces sources (in sinum ecclesiae, in porticibus, in domibus vel in areis ad ecclesiam adiacentibus). Surtout, les deux termes clés de septa et atrium, qui servaient le plus souvent à désigner la zone couverte par le droit d'asile à l'époque médiévale, ne sont pratiquement pas utilisés par Alcuin. Alors que septa est cité deux fois dans ses documents (concile d'Orléans de 541 et Actes de Saint Sylvestre), Alcuin ne l'emploie jamais. Atrium, mentionné pas moins de trois fois dans ses sources (conciles d'Orléans de 511 et 541, et constitution impériale de 431), n'est utilisé par Alcuin qu'une seule fois.

Les nombreuses mentions de l'ecclesia sont évidemment conditionnées par les évènements eux-mêmes, qui se sont déroulés en grande partie dans l'église. Mais nous ne croyons pas que cette seule raison suffise à expliquer le déséquilibre très net entre ecclesia et septa/atrium dans les lettres d'Alcuin. S'il ne s'agit pas non plus d'une volonté délibérée de sa part de valoriser l'ecclesia au détriment de l'atrium, il semble que ce décalage révèle la perception spatiale d'Alcuin, autrement dit son espace vécu. Il y aurait tellement de manières différentes de raconter cette même révolte en employant d'autres mots, et notamment septa et atrium, que la version d'Alcuin nous paraît refléter ses propres catégories spatiales. Ainsi, l'intérêt d'Alcuin ne porte pas tant sur les environs immédiats de l'église que sur l'église elle-même. Si distinction il y a entre un dedans et un dehors, ce n'est pas la limite entre le monde extérieur et l'atrium qui constitue une rupture forte dans l'espace vécu d'Alcuin, mais bien la limite déterminée par les portes de l'église. L'autre limite significative à ses yeux est celle, à l'intérieur de l'église, constituée par le saint des saints biblique, l'espace sacré de l'autel et des reliques : c'est la distinction opérée par Isidore de Séville ${ }^{26}$ entre lieu saint et lieu sacré qui importe à ses yeux, et non pas les catégories spatio-juridiques romaines. Plus que le franchissement de l'atrium, qui ne le choque pas puisqu'il n'en parle même pas, c'est la bousculade des chancels autour de cet espace sacré qui provoque son effroi. Concrètement, l'élément déterminant pour appréhender l'espace concerné par l'asile est l'ecclesia, ce qui contraste singulièrement avec les constitutions romaines qui avaient tenté de cerner plus précisément les limites de l'asile au-delà de la seule église. Pour Alcuin, les lignes de partage essen-

26. M. LAUWERS, Naissance du cimetière..., c. III, Les catégories du sacré, p. 55-56. 
tielles semblent correspondre d'une part aux chancels et, d'autre part, aux portes de l'église, mais pas aux limites de l'atrium ou septa.

\section{La fabrication du droit et le bon exercice de la justice : immunité et droit d'asile}

Nous avons retrouvé un écho de ces préoccupations dans un capitulaire ${ }^{27}$ de Charlemagne de 803 . Les capitula 2 et 3 qui abordent les problèmes posés par l'application de l'immunité et de l'asile nous semblent tout particulièrement liés à ce conflit entre Alcuin et Théodulfe.

Comme la majorité des capitulaires, celui-ci n'a ni suscription, ni adresse, ni date de temps ou de lieu. En revanche, le deuxième manuscrit ${ }^{28}$ de la tradition reconstituée par Alfred Boretius comporte une notice, vraisemblablement contemporaine du capitulaire, qui indique que ce capitulaire a été composée la troisième année de règne de l'imperator Karolus, ce qui a permis de le dater de 803. Cette notice indique également que ces capitula sont destinés à compléter la loi salique, à laquelle il est fait effectivement allusion au chapitre 9 . Il s'agit donc du premier capitulaire du type legibus addenda. D'après François Louis Ganshof ${ }^{29}$, ces capitulaires étaient en préparation dès l'année précédente, à la diète réunie à Aix-la-Chapelle dès l'automne 802. Ils furent élaborés par des membres laïques de la diète tandis que le synodus siégeait à part. Les ducs, les comtes et autres membres de l'aristocratie présents s'assemblèrent, sur ordre de l'empereur, et délibérèrent avec les legislatores au sujet des leges existantes et de leur révision. Ces legislatores étaient des praticiens connaissant les leges, c'est-à-dire les droits régionaux propres aux divers peuples vivant dans l'Empire, principalement les lois des Francs Ripuaires, des Francs Saliens, des Bavarois et des Alamans. Ce capitulaire fut publié dans le missaticum de Paris ${ }^{30}$ par le comte Étienne, qui présida, à l'endroit où siégeait habituellement le mallus, une assemblée composée d'évêques, abbés, comtes et échevins, qui exprimèrent leur consensus et donnèrent confirmation sur le document.

Barbara Rosenwein ${ }^{31}$ a déjà évoqués les capitula 2 et 3 de ce capitulaire afin de montrer que, contrairement à ce que suggérait Élisabeth MagnouNortier la différence entre l'immunité et l'asile était toujours en vigueur à l'époque carolingienne, ce qui paraît justifié.

Le deuxième capitulum stipule que toute personne commettant un crime à l'intérieur d'une immunité doit payer une amende de 600 sous. Le cas est

27. Capitularia ... 1, n 39, p. 111-114.

28. Paris, Bibliothèque nationale de France Richelieu, ms. lat $4995, \mathrm{f}^{\circ} 19 \mathrm{v}^{\circ}$; éd. ibid., $\mathrm{n}^{\circ}$ 39, p. 112, l. 14-21. La datation de cette notice proposée par l'éditeur des MGH est reprise par : F. L. GANSHOF, «Recherches sur les capitulaires », p. 33.

29. F. L. GANSHOF, « Recherches sur les capitulaires", p. 22 et n. 73 , p. 25 n. 88 et p. 53 n. 209 .

30. Ibid., p. 58. Voir la notice du ms. lat. 4995.

31. B. Rosenwein, Negociating Space..., annexe 4, p. 227-228. 
différent si le forfait - peu importe sa nature, crime ou vol - a été perpétré à l'extérieur de l'immunité et si le criminel s'est réfugié ensuite dans une zone immunitaire : à ce moment, le comte doit demander à l'immuniste, qu'il s'agisse d'un évêque, d'un abbé ou de son représentant, de lui livrer le criminel. Si l'immuniste refuse de s'exécuter, il doit payer une amende de 15 sous. S'il persiste à refuser une deuxième fois, il doit s'acquitter d'une peine de 30 sous. S'il s'obstine une troisième fois, il est obligé de payer la totalité de l'amende punissant le crime et le comte aura l'autorisation à partir de là d'entrer dans la zone immunitaire et de se saisir du criminel. De plus, s'il est répondu à la première question du comte que le criminel qui s'était réfugié à l'intérieur de l'immunité a ensuite disparu, l'immuniste doit jurer qu'il n'a pas fait fuir le criminel pour qu'il échappe à la justice du comte. Si quelqu'un tente de s'opposer au comte, qui est entré dans l'immunité pour se saisir du criminel, le comte doit en référer au roi ou au prince afin de juger cette entrave à l'application de la loi : de la même manière qu'un homme commettant un crime dans l'immunité doit payer une amende de 600 sous, celui qui ose résister au comte doit être jugé coupable d'une amende de 600 sous. Ainsi, tous les types de résistances et d'opposition à l'action du comte sont prévus : tout est fait pour permettre au comte de récupérer le criminel réfugié à l'intérieur d'une zone immunitaire.

Le troisième capitulum $^{32}$ prescrit quant à lui que si quelqu'un se réfugie dans une église, cette personne doit bénéficier de la paix dans l'atrium de cette église : il ne lui est pas nécessaire d'entrer dans l'église pour être protégé et personne n'est autorisé à l'extraire de l'atrium par la force. Mais il est permis au réfugié de confesser (confiteri ${ }^{33}$ ) ce qu'il a fait : à partir de là, des hommes compétents (boni homines) doivent le conduire dans un lieu public pour l'examen des faits (ad discussionem in publico).

Plusieurs remarques s'imposent. En premier lieu, il convient de constater, en ce qui concerne le droit d'asile, à quel point la force juridique de l'atrium est réaffirmée au détriment de celle de l'église. Alors qu'Alcuin insistait sur l'ecclesia, celle-ci pose manifestement un problème au pouvoir public car ce capitulum vise à éviter au maximum que les criminels se réfugient dans l'église elle-même. Ce texte témoigne du fait qu'à partir du moment où un homme pénètre dans une ecclesia, il est beaucoup plus difficile de l'en faire sortir, sans doute en raison de la sacralité de l'autel et des reliques. Ce capitulum met donc en exergue la fonction de sas de l'atrium, déjà perceptible dans les sources écrites de l'Antiquité tardive ${ }^{34}$, et qui avait pourtant été négligée par Alcuin. Ainsi, il apparaît clairement que le législateur, qui

32. Capitularia... 1, n ${ }^{\circ}$ 39, cap. 3, p. 113 : Si quis ad ecclesiam confugium fecerit in atrio ipsius ecclesiae pacem habeat, nec sit ei necesse ecclesiam ingredi, et nullus eum inde per vim abstrahere praesumat; sed liceat ei confiteri quod fecit et inde per manus bonorum hominum ad discussionem in publico perducatur.

33. Il s'agit bien ici de la confession, et non pas de l'aveu, puisque le réfugié est autorisé à le faire : il s'agit donc d'un privilège qui lui est reconnu, et non pas d'une obligation juridique.

34. N. Gauthier, "Atria... ", p. 36. 
n'a pas de prise directe à l'intérieur de l'ecclesia, tente de restreindre et confiner le droit d'asile à cet espace transitionnel qu'est l'atrium.

En deuxième lieu, nous pouvons remarquer l'accent mis sur la confession. La confession est considérée comme une première étape vers la réconciliation, mais elle ne peut suffire à elle seule à réparer les torts commis. Une fois la confession célébrée, il n'y a plus de raison de maintenir le fugitif dans l'atrium et celui-ci doit être retiré pacifiquement de cet espace. Le droit d'asile est donc temporaire et ne dure que tant que le réfugié ne s'est pas confessé. Ce capitulum confère ainsi à la confession un rôle majeur dans l'exercice du droit d'asile : c'est parce que le fugitif a été autorisé à se confesser que celui-ci doit accepter de sortir de l'atrium. C'est la confession, et non l'impunité, qui est reconnue comme droit fondamental de tout réfugié. Le droit d'asile est donc réaménagé par ce capitulaire : il est redéfini comme un droit transitionnel, à la fois du point de vue de l'espace (atrium au détriment de l'église), et du point de vue chronologique (jusqu'à la confession).

En troisième lieu, nous remarquons que, comme pour l'immunité, cette procédure vise à permettre la bonne application de la justice. Manifestement, l'intention est de permettre la sortie en douceur du réfugié de la zone protégée par le droit d'asile. À l'instar de l'immunité, le droit d'asile ne doit pas constituer un obstacle à la justice séculière. Ils sont au contraire conçus comme des outils favorisant le dialogue et la réparation du crime dans la mesure où ils permettent de trouver plus facilement un consensus, nécessaire au rétablissement de la paix. Si immunité et asile renvoient effectivement à des notions différentes, la manière d'évoquer ces deux questions est identique dans ce capitulaire. Ces deux capitula visent à trouver les modalités pratiques permettant la résolution du conflit et le rétablissement de la paix, avec un débat public. À chaque fois, il est précisément indiqué de quelle manière les criminels réfugiés dans l'un ou l'autre espace doivent être punis ou récupérés. Il s'agit de faire en sorte que la justice fonctionne correctement, sans être entravée par les privilèges d'exception juridique que sont l'immunité et l'asile. Dans les deux cas, le but est de définir les conditions concrètes d'exercice de la justice afin que les criminels n'utilisent pas ces formes de protection pour échapper au jugement public. Si Charlemagne cherche à ménager les prescriptions du droit ecclésiastique, surtout en ce qui concerne l'asile, il n'est pas question qu'un réfugié détourne ce système pour échapper à la justice. Si le débat public ne peut avoir lieu dans l'atrium lui-même, en raison de la sacralité reconnue à cet espace, il doit avoir lieu à l'extérieur de celui-ci. Tous les cas possibles sont envisagés afin d'éviter le dysfonctionnement judiciaire et les blocages, exactement comme celui entre Alcuin et Théodulphe, qui s'était produit très peu de temps avant l'élaboration de ce capitulaire.

Au total, quatre éléments nous permettent de poser l'hypothèse selon laquelle ces capitula ont été rédigés à cause de ce conflit entre Alcuin et Théodulphe. Premièrement, la proximité chronologique : celle-ci est patente puisque le conflit date de 801-802 et que ce capitulaire était en préparation 
dès 802 , soit immédiatement après l'affaire de ce clerc orléanais. Remarquons que le droit d'asile fait l'objet d'une autre disposition dans le capitulare missorum $^{35}$ de cette même année 803 . Il existe donc bien à cette époque une forte sensibilité aux problèmes posés par les réfugiés. Deuxièmement, la mise en valeur délibérée de l'atrium au détriment de l'ecclesia : ce faisant, ce capitulaire prend acte des faits tourangeaux et cherche à éviter que les complications engendrées par l'entrée du criminel dans l'ecclesia ne se reproduise. Troisièmement, la nouvelle primauté accordée à la confession dans l'application du droit d'asile : sur ce point, le législateur a été sensible au point de vue d'Alcuin, qu'il reformule cependant en inversant l'ordre de priorité entre la justice divine et la justice terrestre. Dans l'esprit de Charlemagne, il convient de permettre le pardon céleste, mais celui-ci ne doit pas se substituer au jugement public. Quatrièmement, l'intention, perceptible aussi bien pour l'immunité que l'asile, d'éviter le blocage de la justice : toutes les situations possibles de contestation du pouvoir public sont énumérées afin de faire face à la réalité sociale. Au total, Charlemagne a manifestement tenu compte des arguments d'Alcuin et reformule les droits d'asile et d'immunité de manière à permettre le bon fonctionnement de la justice publique, qui n'est en aucun cas considérée comme secondaire par rapport à la justice divine. Au contraire, la finalité est bien terrestre, et non pas céleste.

Cela nous amène à formuler un certain nombre de remarques concernant la fabrication du droit à l'époque carolingienne, remarques qui s'inscrivent dans la continuité des travaux de Janet Nelson et Barbara Rosenwein. Cet exemple confirme que la méthode législative carolingienne est fondamentalement pragmatique : la très grande diversité des prescriptions du droit séculier résulte de la fabrication empirique du droit, les capitula étant forgés au cas par cas, selon les questions qui se posaient dans la réalité et qui étaient répercutées lors des plaids. Aucune conception unitaire ne préexiste à l'édification des règles juridiques, au sein desquelles on ne peut déceler aucune volonté de systématiser le droit romain. Au contraire, l'analyse de la reformulation du droit d'asile en 803 montre que le contenu du droit romain, repris à l'époque mérovingienne lors des conciles, a été largement revu et corrigé par Charlemagne dans le but de renforcer la justice publique, quitte à dénaturer ce droit ecclésiastique. À l'époque carolingienne, il n'existe pas non plus de concept abstrait de la loi, ce qui diffère notablement de la législation romaine. Ces discordances amènent Janet Nelson ${ }^{36}$ à présenter les limites de la notion de renaissance carolingienne car, du point de vue juridique, il n'y a aucun retour au droit romain, ni même aucun point commun dans la méthode de fabrication du droit. Notre démarche est également proche de celle de Barbara Rosenwein ${ }^{37}$, qui analyse la réactualisation du concept juridique de l'immunité, dont la signification précise varie

35. Capitularia ... 1, n ${ }^{\circ} 40$, cap. 6, p. 115. Il stipule que tous les fugitifs et les pérégrins doivent être arrêtés afin de connaître leur identité et leur origine.

36. J. L. NELSON, "On the Limits of the Carolingian Renaissance ", p. 56 et 62-63.

37. B. RosenweIn, Negociating space..., p. 9-19. 
selon les contextes. En effet, compte tenu de leurs nouvelles caractéristiques acquises en 803, il est possible de parler d'une reformulation du droit d'asile et de l'immunité, cette réélaboration étant directement liée aux évènements tourangeaux. L'exemple étudié ici prouve que les conventions juridiques sont l'objet d'une renégociation permanente entre les acteurs en fonction de leurs préoccupations et de leur vie concrète.

Ces différents travaux et notre modeste hypothèse sur ce cas tourangeau convergent et gagneraient à être exprimés en terme de paradigme procédural, tel qu'il a été défini par Simona Cerutti ${ }^{38}$. Comme dans le cas de la justice sommaire et du droit naturel à Turin aux XVII ${ }^{\mathrm{e}}$ et XVIII ${ }^{\mathrm{e}}$ siècles, cette affaire montre que les normes peuvent être produites par des pratiques sociales. Ce paradigme procédural est lié à une nouvelle manière d'analyser les comportements sociaux, qui sont réglés par des contraintes qui ne se situent pas seulement au niveau des institutions, mais qui dépendent aussi des propriétés intrinsèques des relations sociales. Simona Cerutti, ainsi que Jacques Revel ${ }^{39}$, proposent d'ancrer les analyses du droit et de l'ordre d'une société dans les processus sociaux, et non pas dans les seules institutions. Une telle conception du rapport normes/pratiques, qui correspond à l'inverse de la conception traditionnelle, conduit à déplacer l'intérêt de la recherche, depuis les institutions préposées au contrôle social, vers les conflits qui se déploient concrètement dans la société et vers les modalités de leurs résolutions. Ainsi, pour comprendre la fabrication du droit, il ne suffit pas d'observer comment les pratiques perçoivent et interprètent les normes, mais il faut surtout analyser les biais par lesquels les pratiques peuvent construire de nouvelles normes. Si Luitpold Wallach a parfaitement confronté pas à pas normes et pratiques, pour s'assurer que les pratiques suivent plus ou moins les normes, il est nécessaire d'analyser les espaces juridiques qui se créent à travers la pratique.

C'est pourquoi les interprétations de Walter Ullmann et François Louis Ganshof concernant la révision des lois nationales et les capitulaires du type legibus addenda ne semblent plus être de mise, ou en tout cas méritent d'être fortement relativisées. Si Walter Ullmann ${ }^{40}$ faisait des capitulaires des instruments du gouvernement impérial carolingien, François Ganshof ${ }^{41}$ distinguait une évolution dans le droit carolingien avant et après le couronnement impérial de Charlemagne. Si, avant 800, les capitulaires n'abordent pas les domaines du droit qui faisaient traditionnellement l'objet des leges (ou droits régionaux), les premiers capitula legibus addenda, qui comportent des révisions sérieuses de ces lois, datent de 803. Outre la proximité chronologique, François Louis Ganshof convoquait deux sources narratives à l'appui de leur hypothèse, la Vita Karoli d'Éginhard ${ }^{42}$ et les

38. S. CERUTTI, "Normes et pratiques... », p. 133-136.

39. J. REVEL, "L'institution et le social ", p. 63-84.

40. W. Ullmann, The Carolingian Renaissance..., p. 30-35.

41. F. L. GANSHOF, "Recherches sur les capitulaires ", p. 96-100.

42. Einhardi vita Karoli Magni, éd. O. Holder-EgGer, c. 29, p. 33. 
Annales de Lorsch ${ }^{43}$, qui suggèrent toutes deux un lien entre l'accession au titre impérial en 800 et la révision de ces leges : c'est le fait d'avoir été sacré empereur qui aurait incité Charlemagne, une fois devenu le successeur de Constantin et Théodose, à corriger le droit " national " traditionnel, ce droit ancien étant considéré jusque-là comme un héritage sacré et intouchable. Après 800 , Charlemagne aurait pris une conscience très forte du caractère extraordinaire et unique de sa nouvelle dignité, prise de conscience à laquelle les " impérialistes " de son entourage auraient fortement contribué. En 802, au cours des mois de méditation et de conversation avec ses conseillers à Aix-la-Chapelle, se serait développée et ancrée en lui la conscience de ce qu'il était désormais la source du droit, soit un législateur au plein sens du terme, comme l'avait été ses illustres prédécesseurs, les empereurs romains chrétiens.

Cette théorisation, aussi séduisante soit-elle, ne semble pas résister à l'examen des faits précis contenus dans ces capitulaires. La manière dont ils ont été rédigés montre que, loin d'être préconçus comme des instruments du nouveau gouvernement impérial, ils répondent à une question concrète, issue du conflit entre Alcuin et Théodulphe : comment concilier les formes ecclésiastiques de protection que sont l'asile et l'immunité et le bon fonctionnement de la justice publique? Les indications de ces deux sources narratives témoignent peut-être de l'émergence d'une nouvelle conscience impériale chez ces auteurs ecclésiastiques, mais certainement pas d'une nouvelle manière de fabriquer le droit chez Charlemagne. Le but de Charlemagne n'est pas d'imposer une norme ecclésiastique à l'ensemble de la société, mais de répondre aux problèmes qui se posent : bien au contraire, il affirme que la justice céleste ne constitue qu'un préalable à la justice terrestre. Il ne faut donc pas tout imputer au mouvement culturel de la renaissance carolingienne. En l'occurrence, Charlemagne ne cherche pas à appliquer un modèle, mais à répondre à une demande née de la praxis des acteurs. Si ce clerc orléanais n'était pas venu se réfugier à Saint-Martin, et si Alcuin n'avait pas ouvertement pris sa défense, ces capitula n'auraient peut-être pas vu le jour.

Les conséquences juridiques induites par cette affaire du réfugié orléanais témoignent de l'intérêt du paradigme procédural pour mieux comprendre la fabrication du droit à l'époque carolingienne. Ce conflit montre que l'excessive utilisation de la notion de renaissance carolingienne nuit plus qu'autre chose à la bonne compréhension des réalités politiques et sociales carolingiennes. Non seulement, la loi romaine n'est pas considérée comme un modèle auquel il faudrait revenir, mais c'est surtout suite au conflit entre Alcuin et Théodulphe que Charlemagne réélabore sa propre loi pour améliorer l'exercice de la justice. Nous ne voyons rien qui s'appa-

43. Annales Laureshamenses, éd. G.-H. Pertz, MGH SS, 1, année 802, cap. 35, p. 3839. Cette interprétation a été reprise dernièrement par: Ph. DEPREUX, "Ambitions et limites des réformes culturelles...", p. 734-736. 
rente à une nouvelle conception du monde elle-même liée au sacre impérial. Ces lois carolingiennes ne sont pas des théories sorties toutes droites de la tête de l'empereur, telle Athéna sortie casquée de la tête de Zeus. Les normes législatives paraissent au contraire issues de conflits concrets comme cette affaire. La construction de ces nouvelles normes juridiques à partir de la pratique, certes plus difficile à percevoir que l'action volontaire d'un homme politique, nous fait douter du bien fondé de la généralisation de la notion de "renaissance carolingienne " à tous les domaines de l'écrit, et singulièrement à celui du droit.

\section{RESUME}

Le conflit qui a opposé en 801-802 Alcuin à Théodulfe, à propos d'un clerc réfugié dans la basilique de Saint-Martin, éclaire les pratiques juridiques et culturelles de cette époque. Grâce à la confrontation de deux types de sources - quatre lettres d'Alcuin et le capitulaire de 803 - les différents aspects de ce conflit permettent de formuler quelques remarques sur la fabrication du droit, ainsi que sur la notion de renaissance carolingienne, qui ne semble pas devoir être généralisée à tous les domaines de l'écrit. Les relations entre normes juridiques et pratiques sociales seront envisagées dans l'autre sens que celui habituellement analysé, en observant de quelle manière une norme peut être produite à partir de la pratique sociale, c'est-à-dire comment l'immunité et le droit d'asile sont réélaborés à partir de ce conflit.

\section{ABSTRACT}

The conflict that opposed in 801-802 Alcuin to Théodulfe, by the way of a clerk refugee in the basilica of Saint-Martin, highlights the legal and cultural practices of this time. Thanks to the crossing of two types of sources - four letters of Alcuin and the capitulary of 803 - the different aspects of this conflict allow to formulate some remarks on the making of the Right, as well as on the Carolingian Rebirth notion, that doesn't seem to be generalized to all domains of the writing. Relations between social practice and legal norms will be considered in the opposite direction that the one usually analysed, taking in consideration how a norm can be produced from the social practice, that means how the expression of immunity and right of asylum in the capitulary of 803 comes from this conflict. 
